\title{
Transmission Strategies for Correlated MIMO Links with Imperfect Channel Estimates
}

\author{
Semih Serbetli and Aylin Yener \\ Electrical Engineering Department \\ The Pennsylvania State University \\ University Park, PA 16802 \\ serbetli@psu.edu yener@ee.psu.edu
}

\begin{abstract}
We investigate the transmit precoder design problem for a multiple input multiple output (MIMO) link with correlated receive antennas, considering the effect of channel estimation. We propose transceiver structures that are optimum in the sense of minimizing the total MSE and distributing the total MSE equally among the parallel data streams. We also investigate the problem of how the correlated MIMO link should distribute its total available power between power expended for channel estimation versus data transmission. The optimum power allocation problem between the training sequences for channel estimation and data transmission for the correlated MIMO link is shown to have a unique solution, that is different than the uncorrelated case. The performance of the system is observed to be more sensitive to transceiver design rather than the power allocation. The results demonstrate that the correlation structure of the MIMO link has a profound effect on the performance, and that the transceiver optimization should be done by taking both the correlation and the channel estimation process into account.
\end{abstract}

\section{INTRODUCTION}

Recent studies indicate that using multiple antennas at the transmitter and receiver can dramatically improve the performance of wireless communication systems [1]. There has been considerable research in exploiting the space dimension through transmit diversity, space-time coding and spatial multiplexing for MIMO systems [2], [3].

Performance of a MIMO system is highly dependent on the channel state information (CSI) available at both the transmitter and the receiver side. Hence, estimation of the channel at the receiver side, and feedback of this information to the transmitter side have significant impact on the performance. In the absence of CSI at the transmitter side, multiple antennas can be used for spatial multiplexing [3], or for space-time coding [2]. The effect of receiver side channel estimation on such schemes is analyzed in [2], [4]. Spatial multiplexing can significantly benefit from transmit precoding when CSI is available at both transmitter and receiver side [5].

In order to design the "right" transmission strategy, the MIMO channel has to be estimated at the receiver, and in turn should be fedback to the transmit side. To that end, the design of optimum training sequences for estimation of uncorrelated and correlated MIMO channels are studied in [4], [6]. Also considered in detail, in references [7]-[9], are optimal transmission strategies with imperfect CSI at the transmitter side. We must note that all of these approaches optimize the transmission strategy for a given channel estimation process, and assume perfect CSI at the receiver side.

In practice, it is likely that the total transmission power budget would be limited for the MIMO system. Hence, it is meaningful to ask what fraction of the resources should be devoted to estimation versus actual data transmission. Towards that end, optimum training sequences and power allocation among the training sequences and data transmission are found for BLAST transmission in [4]. Reference [4] considered a lower bound of the sum capacity as the performance metric, and assumed uncorrelated MIMO links. Reference [10], on the other hand, considered the total MSE as the performance metric for uncorrelated MIMO systems, and found that the optimal power allocation to be identical to the one found in [4].

In this paper, we consider the uplink of a MIMO system, and extend the work in [10] for the case of correlated receive antennas. The existence of correlation between the receive antennas is a likely scenario due to the fact that fewer local scatterers exist near the base station antennas. We address the case where the receiver estimates the channel, and that both the transmitter and the receiver have access to the same imperfect CSI. Throughout the paper, we use the total mean squared error (MSE) as our performance metric. We investigate the joint effect of channel estimation process and the correlation among the receiver antennas on the design of the precoder and the decoder for the MIMO system, with the objective of minimizing the MSE, such that each symbol is transmitted with an equal MSE performance. We derive the optimal precoder and decoder structures, and show that both the correlation, and the channel estimation process should be taken into account for transceiver optimization. Next, we consider the problem optimum sharing of resources between the process of channel estimation and data transmission. We consider the total power as the limited resource, [4], [10], but consider the more general problem of power allocation between the channel estimation and data transmission for the correlated MIMO link. We show that, given the coherence time of the channel, there is a unique solution to the optimum power allocation problem. More importantly, we show that this solution is different than the uncorrelated case. The results also demonstrate that the system performance is more sensitive to the transceiver design rather than the power allocation. 


\section{System Model And Performance Metric}

We consider a communication link consisting of $N_{T}$ transmitter antennas and $N_{R}$ receive antennas. The transmitter multiplexes $M$ data streams through $N_{T}$ transmit antennas employing an $N_{T} \times M$ linear transmitter $\mathbf{F}$ in one symbol period. Similar to the notation in [5], the received vector is

$$
\mathbf{r}=\mathbf{H F s}+\mathbf{n}
$$

where, $\mathbf{s}$ is the $M \times 1$ symbol vector, $\mathbf{H}$ is a realization of the $N_{R} \times N_{T}$ random matrix of complex channel gains with correlated receive antennas, $\mathcal{H}$, with $E\left\{\mathcal{H} \mathcal{H}^{\dagger}\right\}=N_{T} \mathbf{C}_{R X}$, and uncorrelated transmit antennas, $E\left\{\mathcal{H}^{\dagger} \mathcal{H}\right\}=\gamma \mathbf{I}$, where $(\cdot)^{\dagger}$ denotes the hermitian of a vector or matrix. $\mathbf{n}$ is the zero mean complex Gaussian noise vector with $E\left[\mathbf{n n}^{\dagger}\right]=\sigma^{2} \mathbf{I}$.

We assume that the channel is flat fading with coherence time of $\left(L_{t r}+L_{d}\right)$ symbols where $L_{t r}$ symbol intervals are dedicated to training sequences, and the remaining $L_{d}$ to data transmission. Reference [6] showed that orthogonal training sequences are optimum for estimating MIMO channels with correlated receive antennas. Hence, throughout this paper, we assume that orthogonal training sequences are used for channel estimation. The power available to the system for the entire interval is $P_{\text {total }}$ where $P_{t r}$ portion of it is used for the transmission of the training sequences and the remaining portion is distributed equally among the $L_{d}$ symbols. Thus, the precoder should be designed with the power constraint $\operatorname{tr}\left\{\mathbf{F F}^{\dagger}\right\} \leq P_{s}=\left(P_{\text {total }}-P_{t r}\right) / L_{d}$. First, we analyze the effect of channel estimation on the design of linear transmitter (precoder) and receiver (decoder) for a given power allocation. Later in the paper, we investigate the power allocation problem among the training sequences for the channel estimation and the actual data transmission.

Throughout the paper, it is assumed that the receiver obtains the ML estimate of the MIMO channel $\hat{\mathcal{H}}=\mathcal{H}+\mathcal{X}$ that is fedback to the transmitter via an error-free feedback channel. Following the ML estimate model of the MIMO channel in [4], [6], when optimal training sequences of length $L_{t r} \geq N_{R}$ are used, $\mathcal{X}$ is a random matrix with i.i.d. complex Gaussian entries having $C N\left(0, \sigma_{e}^{2}=\frac{\left(\sigma^{2} N_{T}\right)}{P_{t r}}\right)$ and is independent of the MIMO channel $\mathcal{H}$. Recall that $\mathcal{H}$ is a complex Gaussian random matrix with $E\left\{\mathcal{H} \mathcal{H}^{\dagger}\right\}=N_{T} \mathbf{C}_{R X}=N_{T} \mathbf{U}_{R X} \Lambda \mathbf{U}_{R X}^{\dagger}$. Hence, the ML channel estimate can be represented as $\mathcal{H}=$ $\mathbf{U}_{R X} \Lambda^{1 / 2} \mathcal{H}_{\mathcal{W}}+\mathcal{X}$ where the elements of $\mathcal{H}_{\mathcal{W}}$ are i.i.d. with $C N(0,1)$. It can be easily seen that the distribution of the $i$ th column of $\mathcal{H}, \mathcal{H}_{i}$, for a given estimate $\hat{\mathcal{H}}=\hat{\mathbf{H}}$ is $C N\left(\left(\mathbf{H}_{\mu}\right)_{i}, \mathbf{C}_{\mathcal{H} \mid \hat{\mathbf{H}}}\right)$ with

$$
\begin{gathered}
E\left\{\mathcal{H}_{i} \mid \hat{\mathcal{H}}=\hat{\mathbf{H}}\right\}=\left(\mathbf{H}_{\mu}\right)_{i}=\mathbf{U}_{R X} \Lambda\left(\Lambda+\sigma_{e}^{2} \mathbf{I}\right)^{-1} \mathbf{U}_{R X}^{\dagger} \hat{\mathbf{H}}_{i} \\
E\left\{\mathcal{H}_{i} \mathcal{H}_{i}^{\dagger} \mid \hat{\mathcal{H}}=\hat{\mathbf{H}}\right\}=\mathbf{C}_{\mathcal{H} \mid \hat{\mathbf{H}}}=\sigma_{e}^{2} \mathbf{U}_{R X} \Lambda\left(\Lambda+\sigma_{e}^{2} \mathbf{I}\right)^{-1} \mathbf{U}_{R X}^{\dagger}
\end{gathered}
$$

where $\hat{\mathbf{H}}_{i}$ is the $i$ th column of $\hat{\mathbf{H}}$. Observe that from the receiver's perspective, the actual channel is a random MIMO channel with mean $\mathbf{H}_{\mu}=\left[\left(\mathbf{H}_{\mu}\right)_{1},\left(\mathbf{H}_{\mu}\right)_{2} \ldots\left(\mathbf{H}_{\mu}\right)_{N_{T}}\right]$ and; $E\left\{\mathcal{H} \mathcal{H}^{\dagger} \mid \hat{\mathcal{H}}=\hat{\mathbf{H}}\right\}=N_{T} \mathbf{C}_{\mathcal{H} \mid \hat{\mathbf{H}}}$ and $E\left\{\mathcal{H}^{\dagger} \mathcal{H} \mid \hat{\mathcal{H}}=\hat{\mathbf{H}}\right\}=v \mathbf{I}$.

Let us denote the $M \times N_{R}$ linear receiver by $\mathbf{G}$; the decision statistic $\mathbf{y}$, for a channel realization $\mathbf{H}$, is then given by

$$
\mathbf{y}=\mathbf{G H F s}+\mathbf{G n}
$$

For a given $\hat{\mathbf{H}}$, the total MSE can be expressed as

$$
\operatorname{MSE}_{\hat{\mathbf{H}}}=E\left[\operatorname{tr}\left\{\mathbf{F}^{\dagger} \mathcal{H}^{\dagger} \mathbf{G}^{\dagger} \mathbf{G} \mathcal{H} \mathbf{F}-\mathbf{F}^{\dagger} \mathcal{H}^{\dagger} \mathbf{G}^{\dagger}-\mathbf{G} \mathcal{H} \mathbf{F}+\mathbf{I}+\sigma^{2} \mathbf{G G}^{\dagger}\right\}\right]
$$

where $\operatorname{tr}\{\mathbf{A}\}$ denotes the trace of matrix $\mathbf{A}$.

Total MSE minimization by choosing the transmitters and receivers has been studied for uncorrelated MIMO links with exact channel state information in [5]. In practice, the CSI available to the transmitter and receiver would not be perfect. In addition, it is meaningful to consider a system where fairness is facilitated by ensuring that each symbol experiences equal MSE. In Section III, we pose the problem of minimizing the total MSE for a given ML estimate of the correlated MIMO channel, $\mathrm{MSE}_{\hat{\mathbf{H}}}$, considering fairness among the parallel data streams, and construct the optimum transceiver structure.

Note that when the optimum linear transmitter and receiver are used at each realization, the total MSE over all channel realizations and estimates, MSE, can be expressed as

$$
\operatorname{MSE}=E\left[\min _{\{\mathbf{F}, \mathbf{G}\}} \operatorname{MSE}_{\hat{\mathbf{H}}}\right]
$$

We will use (6) as the performance metric in Section IV.

\section{Optimum TRANSCEIVER StRUCTURE}

Our aim in this section is to find the optimum transceiver structure in the sense of minimizing the total MSE and distributing the total MSE equally among the parallel data streams, while taking into account the effect of ML channel estimation. Formally, the optimization problem is

$$
\begin{gathered}
\min _{\{\mathbf{F}, \mathbf{G}\}} \quad \operatorname{MSE}_{\hat{\mathbf{H}}}=\operatorname{tr}\{\mathbf{B}\} \\
\text { s.t. } \operatorname{tr}\left\{\mathbf{F} \mathbf{F}^{\dagger}\right\} \leq P_{s} ; \\
\mathbf{B}=E \mathbf{F S E}_{1}^{\dagger} \mathcal{H}^{\dagger} \mathbf{G}^{\dagger} \mathbf{G} \mathcal{H} \mathbf{F}-\mathbf{F S E}_{2}^{\dagger} \mathcal{H}^{\dagger} \mathbf{G}^{\dagger}-\mathbf{G} \mathcal{H} \mathbf{F}+\mathbf{I}+\sigma^{2} \mathbf{G G}^{\dagger}
\end{gathered}
$$

and $\mathrm{MSE}_{i}$ is the individual MSE of data stream $i$, and is the $(i, i)$ th entry of $\mathbf{B}$.

Recall that from the receiver's perspective the MIMO channel can be modelled as $\mathcal{H}=\mathbf{H}_{\mu}+\mathbf{C}_{\mathcal{H} \mid \hat{\mathbf{H}}}^{1 / 2} \mathcal{Z}$ where $\mathcal{Z}$ is a random matrix with i.i.d. complex Gaussian entries of $C N(0,1)$. Inserting the expression above for $\mathcal{H}$ in (5) and taking the expectation with respect to $\mathcal{Z}, \operatorname{MSE}_{\hat{\mathbf{H}}}$ is

$$
\operatorname{tr}\left\{\mathbf{F}^{\dagger} \mathbf{H}_{\mu}^{\dagger} \mathbf{G}^{\dagger} \mathbf{G} \mathbf{H}_{\mu} \mathbf{F}-\mathbf{F}^{\dagger} \mathbf{H}_{\mu}^{\dagger} \mathbf{G}^{\dagger}-\mathbf{G H}_{\mu} \mathbf{F}+\mathbf{I}+\mathbf{G C}_{c n} \mathbf{G}^{\dagger}\right\}
$$

where $\mathbf{C}_{c n}=\sigma^{2} \mathbf{I}+\operatorname{tr}\left\{\mathbf{F} \mathbf{F}^{\dagger}\right\} \mathbf{C}_{\mathcal{H} \mid \hat{\mathbf{H}}}$. Observe that the total MSE in (9) has the same form of the total MSE expression of a MIMO system with a channel matrix $\mathbf{H}_{\mu}$ and a colored noise factor with covariance $\mathbf{C}_{c n}$.

Let us now consider the minimization of the total MSE in terms of the precoder and decoder. The first order condition with respect to the linear receiver (decoder) results in the wellknown MMSE receiver $\mathbf{G}=\mathbf{F}^{\dagger} \mathbf{H}_{\mu}^{\dagger}\left(\mathbf{C}_{c n}+\mathbf{H}_{\mu} \mathbf{F} \mathbf{F}^{\dagger} \mathbf{H}_{\mu}^{\dagger}\right)^{-1}$. Using MMSE receivers, the total MSE can be reformulated as

$$
\mathrm{MSE}_{\hat{\mathbf{H}}}=M-N_{R}+\operatorname{tr}\left\{\mathbf{T}^{-1}\right\}
$$


where $\mathbf{T}=\mathbf{I}+\mathbf{H}_{e} \mathbf{F F}^{\dagger} \mathbf{H}_{e}^{\dagger}$ with $\mathbf{H}_{e}=\mathbf{C}_{c n}^{-1 / 2} \mathbf{H}_{\mu}$. Notice that the optimum linear transmitter and receiver set that minimizes the total MSE is not unique, and any linear transmitter that achieves the same covariance $\mathbf{F F}^{\dagger}=\widetilde{\mathbf{F}} \widetilde{\mathbf{F}}^{\dagger}$ achieves the same total MSE. Specifically, all optimum linear transmitters are in the form of $\left\{\mathbf{F}_{o p t}^{\star}\right\}=\mathbf{F}_{o p t} \mathbf{U}^{\dagger}$ where $\mathbf{U}$ is an arbitrary $M \times M$ matrix satisfying $\mathbf{U}^{\dagger} \mathbf{U}=\mathbf{I}$.

It is evident that the minimum total MSE without any constraints lower bounds the minimum total MSE with fairness constraints. However, as is noted below, the optimum transceiver structure with fairness constraints lies in the set of transmitters that yield the unconstrained minimum total MSE for a special structure of $\mathbf{U}$.

For $M \leq \operatorname{rank}\left(\mathbf{H}_{e}\right)$, reference [5] suggests that one possible optimum linear transmitter without fairness constraints, is in the form of $\mathbf{F}_{\text {opt }}=\mathbf{V}_{e} \mathbf{Q}_{f}$ where $\mathbf{V}_{e}$ is an $N_{T} \times M$ orthogonal matrix that has columns as the eigenvectors of the largest $M$ eigenvalues of $\mathbf{H}_{e}^{\dagger} \mathbf{H}_{e}=\left[\begin{array}{cc}\mathbf{V}_{e} & \tilde{\mathbf{V}}_{e}\end{array}\right]\left[\begin{array}{cc}\Lambda_{e} & 0 \\ 0 & \tilde{\Lambda}_{e}\end{array}\right]\left[\begin{array}{c}\mathbf{V}_{e}^{\dagger} \\ \tilde{\mathbf{V}}_{e}^{\dagger}\end{array}\right]$ where $\Lambda_{e}$ is a diagonal matrix containing the largest $M$ eigenvalues arranged in a decreasing order from top-left to bottom-right, and $\mathbf{Q}_{f}=\left(\mu^{-1 / 2} \Lambda_{e}^{-1 / 2}-\Lambda_{e}^{-1}\right)_{+}^{1 / 2}$ is a diagonal matrix with $\mu$ factor satisfying the power constraint, and $(.)_{+}=$ $\max (0,$.$) . If \hat{\mathbf{H}}$ were the perfect CSI, then the optimum linear transmitter would be in the form of $\hat{\mathbf{F}}_{o p t}=\hat{\mathbf{V}}_{e} \hat{\mathbf{Q}}_{f}$ with $\hat{\mathbf{Q}}_{f}=\left(\hat{\mu}^{-1 / 2} \hat{\Lambda}_{e}^{-1 / 2}-\hat{\Lambda}_{e}^{-1}\right)_{+}^{1 / 2} \cdot \hat{\mathbf{V}}_{e}, \hat{\Lambda}_{e}$, and $\hat{\mathbf{Q}}_{f}$ are the corresponding eigenvectors, eigenvalues and power allocation for $1 / \sigma^{2} \hat{\mathbf{H}}^{\dagger} \hat{\mathbf{H}}$, respectively. Observe that, in this case, the optimum linear transmitter results in transmitting in both different eigenmodes and power allocation. It can also be shown that the optimum transmit covariance matrix, i.e., $\mathbf{F}_{\text {opt }} \mathbf{F}_{\text {opt }}^{\dagger}$ for $M=\operatorname{rank}\left(\mathbf{H}_{e}\right)$ is also the optimum covariance matrix for $M>\operatorname{rank}\left(\mathbf{H}_{e}\right)$ [10]. Specifically, all optimum linear transmitters are in the form of $\mathbf{F}_{\text {opt }}=\mathbf{V}_{e} \mathbf{Q}_{f} \mathbf{U}^{\dagger}$ where $\mathbf{U}^{\dagger} \mathbf{U}=\mathbf{I}$. Thus, the total MSE of each data stream can be expressed by the diagonal entries of

$\mathbf{B}=\mathbf{I}-\mathbf{F}_{\text {opt }}^{\dagger} \mathbf{H}_{\mu}^{\dagger}\left(\mathbf{C}_{c n}+\mathbf{H}_{\mu} \mathbf{F}_{\text {opt }} \mathbf{F}_{\text {opt }}{ }^{\dagger} \mathbf{H}_{\mu}^{\dagger}\right)^{-1} \mathbf{H}_{\mu} \mathbf{F}_{\text {opt }}=\mathbf{U D U}^{\dagger}$

where $\mathbf{D}$ is a diagonal matrix.

Now, consider a MIMO system where equal MSE values for each symbol is required. Since the diagonal entries of $\mathbf{B}$, the achieved MSE of each data stream, are desired to be equal, a $\mathbf{U}$ that results in a $\mathbf{B}$ matrix with equal diagonal entries is needed. Reference [9], [11] suggest that the discrete Fourier transform matrix or the Hadamard matrix (when $M$ is a power of 2) provides a simple construction of this special matrix.

\section{Power Allocation Trade-OFFs}

\section{A. Optimum Power Allocation}

It is evident from the preceding discussion in this paper, as well as several other references, e.g., [4], [12], that the availability of an accurate channel estimate has a substantial impact on the performance of a MIMO link. Therefore, it makes sense to devote some part of system resources to the channel estimation process if in turn the gain in performance is worth the effort. In practice, it is likely that, in a given interval, where the channel is likely to be static, the link would operate with a limited total budget. It is then meaningful to ask what fraction of this total power budget should be expended on the transmission of training sequences that are used in estimating the channel, versus the transmission of actual data.

In this section, we investigate the optimum power allocation problem between training and data transmission using total MSE as the performance metric. Recall that for a given channel estimate, minimizing the total MSE, $\mathrm{MSE}_{\hat{\mathbf{H}}}$, is minimizing $M-N_{R}+\operatorname{tr}\left\{\left(\mathbf{I}+\mathbf{C}_{c n}^{-1 / 2} \mathbf{H}_{\mu} \mathbf{F} \mathbf{F}^{\dagger} \mathbf{H}_{\mu}^{\dagger} \mathbf{C}_{c n}^{-1 / 2}\right)^{-1}\right\}$ where $\mathbf{H}_{\mu}$ is a realization of the random matrix $\tilde{\mathcal{H}}=\mathbf{U}_{R X} \Lambda(\Lambda+$ $\left.\sigma_{e}^{2} \mathbf{I}\right)^{-1} \mathbf{U}_{R X}^{\dagger} \hat{\mathcal{H}}$ with $\hat{\mathcal{H}}=\mathbf{U}_{R X} \Lambda^{1 / 2} \mathcal{H}_{W}+\mathcal{X}$. Thus, minimizing the total MSE, given that we use the optimum precoder, over all channel realizations and estimates is equivalent to

$$
\min E\left[\min _{\mathbf{F}} \operatorname{tr}\left\{\left(\mathbf{I}+\mathbf{C}_{c n}^{-1 / 2} \tilde{\mathcal{H}} \mathbf{F} \mathbf{F}^{\dagger} \tilde{\mathcal{H}}^{\dagger} \mathbf{C}_{c n}^{-1 / 2}\right)^{-1}\right\}\right]
$$

Observe that we can express $\hat{\mathcal{H}}=\mathbf{U}_{R X}\left(\Lambda+\sigma_{e}^{2} \mathbf{I}\right)^{1 / 2} \Upsilon$ where $\Upsilon$ is a random matrix with complex Gaussian entries with $C N(0,1)$ and $\operatorname{tr}\left\{\mathbf{F} \mathbf{F}^{\dagger}\right\}=P_{s}$. Inserting $\mathbf{C}_{c n}=\sigma^{2} \mathbf{I}+$ $\operatorname{tr}\left\{\mathbf{F} \mathbf{F}^{\dagger}\right\} \mathbf{C}_{\mathcal{H} \mid \hat{\mathbf{H}}}$, and the expressions for $\tilde{\mathcal{H}}$ and $\hat{\mathcal{H}}$ given above, in (12) we can show that

$\min \mathrm{MSE} \equiv \min E\left[\min _{\mathbf{F}} \operatorname{tr}\left\{\left(\mathbf{I}+\Delta^{1 / 2} \Upsilon\left(\frac{1}{P_{s}} \mathbf{F F}^{\dagger}\right) \Upsilon^{\dagger} \Delta^{1 / 2}\right)^{-1}\right\}\right]$

where $\Delta=P_{s} \Lambda^{2}\left(\sigma^{2}\left(\Lambda+\sigma_{e}^{2} \mathbf{I}\right)+P_{s} \sigma_{e}^{2} \Lambda\right)^{-1} . \Delta$ is a diagonal matrix with $\Delta_{i i}=\frac{\Lambda_{i}^{2} P_{s}}{\sigma^{2}\left(\Lambda_{i}+\sigma_{e}^{2}\right)+\sigma_{e}^{2} \Lambda_{i} P_{s}}$ as the $i$ th diagonal entry. Thus, the expressions $\left\{\Delta_{i i}\right\}$ act like the effective SNRs of each virtual receive antenna.

We have the following relationship between data transmission power and power dedicated to the training sequences

$$
P_{s} L_{d}+P_{t r}=P_{\text {total }}
$$

Defining $\alpha$ to be the fraction of the total power devoted to data transmission, i.e., $\alpha=\frac{P_{s} L_{d}}{P_{\text {total }}} \quad 0 \leq \alpha \leq 1$, and

$$
c_{i}=\frac{\left(N_{T}-L_{d}\right) P_{\text {total }} \Lambda_{i}}{L_{d}\left(N_{T} \sigma^{2}+P_{\text {total }} \Lambda_{i}\right)} ; d_{i}=\frac{P_{\text {total }}^{2} \Lambda_{i}{ }^{2}}{\sigma^{2} L_{d}\left(\Lambda_{i} P_{\text {total }}+N_{T} \sigma^{2}\right)}
$$

the effective SNR of each virtual receive antenna can be expressed as

$$
\Delta_{i i}(\alpha)=\frac{d_{i} \alpha(1-\alpha)}{c_{i} \alpha+1}
$$

and we have the following Lemma for $\left\{\Delta_{i i}\right\}$.

Lemma 1: $\Delta_{i i}(\alpha)$ is concave over the interval $[0,1]$, and the optimum $\alpha$ maximizing $\Delta_{i i}(\alpha)$ is given by

$$
\alpha_{i}^{\star}=\begin{array}{ll}
\frac{-1+\sqrt{1+c_{i}}}{c_{i}}, & \text { for } N_{T}>L_{d} ; \\
\frac{\frac{1}{2},}{\frac{-1+\sqrt{1+c_{i}}}{c_{i}}}, & \text { for } N_{T}=L_{d} ; \\
& \text { for } N_{T}<L_{d} ;
\end{array}
$$

where $c_{i}$ is given in (15).

Defining the set of diagonal SNR matrices, $\Psi$, with $\Omega \in \Psi$ if $\Delta(\alpha)-\Omega \geq \mathbf{0}$ for $\exists \alpha \in[0,1]$, the optimum power allocation problem, $\min _{0<\alpha<1}$ MSE, is equivalent to

$$
\min _{\Omega \in \Psi} E\left[\min _{\mathbf{F}} \operatorname{tr}\left\{\left(\mathbf{I}+\Omega^{1 / 2} \Upsilon\left(\frac{1}{P_{s}} \mathbf{F F}^{\dagger}\right) \Upsilon^{\dagger} \Omega^{1 / 2}\right)^{-1}\right\}\right]
$$




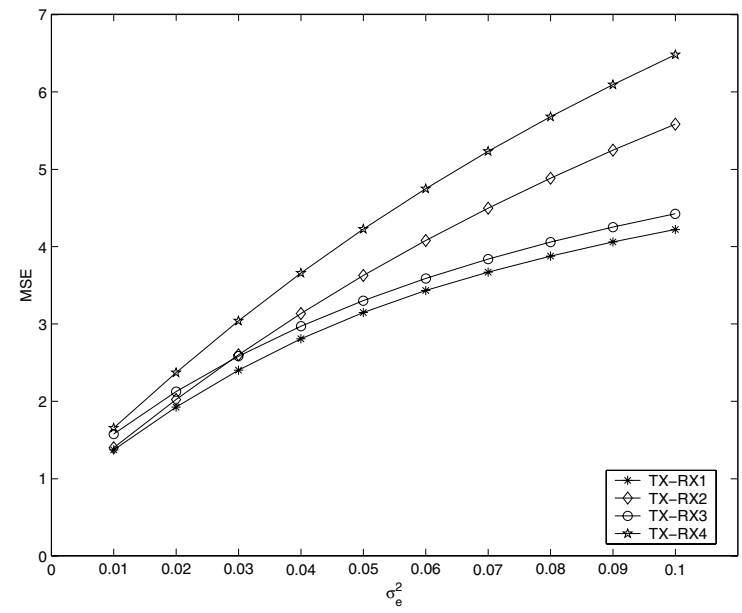

Fig. 1. MSE vs $\sigma_{e}^{2}$ performance for $8 \times 8$ MIMO system

It can be easily seen that the total MSE function is strictly convex over the SNR matrix, $\Omega$. In addition, the SNR matrix set, $\Psi$ is a convex set. Thus, there exists a unique optimum power allocation scheme minimizing the total MSE, and we have the following theorem that guarantees the existence of the global minimizer for the optimum power allocation problem.

Theorem 1: The global minimizer $\alpha_{\text {opt }}$ of (18) is unique and $\alpha_{\text {opt }} \in\left[\alpha_{\min }=\min _{i} \alpha_{i}^{\star}, \alpha_{\max }=\max _{i} \alpha_{i}^{\star}\right]$.

The proofs are omitted here due to space limitations and can be found in [13]. Lastly, we note that $\alpha_{\text {opt }}$ can be found by a variety of iterative algorithms [14].

\section{B. Observations}

The value of $\alpha_{\text {opt }}$ depends on the number of transmit antennas, and the length of the time interval used for symbol transmission. We observe that when $N_{T}>L_{d}$, then all $c_{i}$ 's are positive resulting in all $\alpha_{i}^{\star}$ to be less than $1 / 2$. Thus, $\alpha_{\text {opt }}$ lies in the range of $\left[0, \frac{1}{2}\right)$. This result suggests allocating more power to training for such systems with large number of transmit antennas.

When $N_{T}<L_{d}$, then the range of $\alpha_{i}^{\star}$ is $\left(\frac{1}{2}, 1\right]$ resulting $\alpha_{\text {opt }}$ to be in $\left(\frac{1}{2}, 1\right]$. This implies that when the data transmission interval is much larger than the number of transmit antennas, a significant portion of the system power should be allocated to symbol transmission rather than the estimation process.

For the case where $N_{T}=L_{d}$, all $\alpha_{i}$ 's are $1 / 2$ resulting $\alpha_{\text {opt }}=1 / 2$. That is, the available power should be allocated equally between training and data transmission.

When, we consider the optimum power allocation for high and low SNR cases, the following power distribution schemes and effective SNRs are observed:

For high SNR, i.e., $P_{\text {total }} \rightarrow \infty$, we have $c_{i}=$ $\frac{N_{T}-L_{d}}{L_{d}} \quad \forall i=1,2, \ldots, N_{R}$ resulting $\alpha_{\text {opt }}=\frac{\sqrt{L_{d}}}{\sqrt{N_{T}}+\sqrt{L_{d}}}$.

For low SNR, i.e., $P_{\text {total }} \rightarrow 0$, we have $c_{i} \stackrel{=}{=} \forall i=$ $1,2, \ldots, N_{R}$ resulting $\alpha_{\text {opt }}=\frac{1}{2}$

Observe that the optimum power allocation for both high and low SNR regimes does not depend on the correlation of the receive antennas, resulting in the same asymptotic optimal power allocation as the uncorrelated case. However, outside of

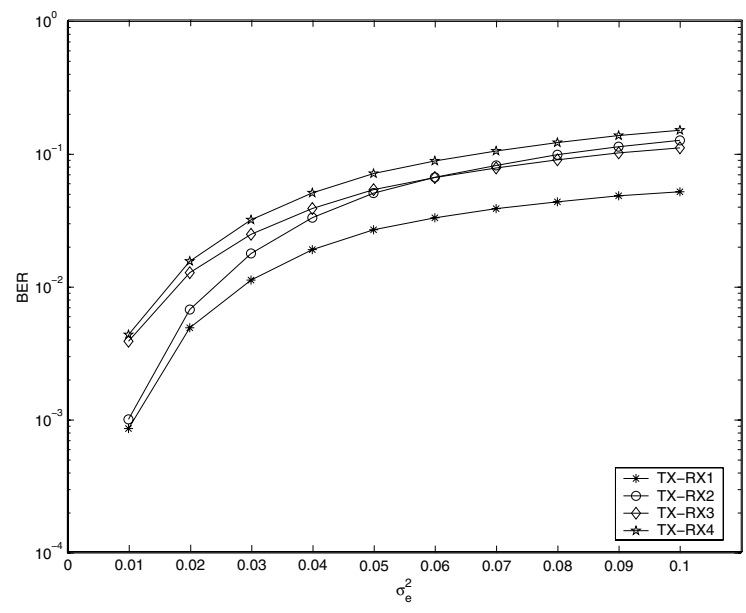

Fig. 2. BER vs $\sigma_{e}^{2}$ performance for $8 \times 8$ MIMO system

these asymptotic regions, the resulting power allocation differs from the uncorrelated case as evidenced next.

\section{NumericAl RESUlts}

In this section, we present numerical results related to the performance of the proposed transceiver structures and optimum power allocation for channel estimation and data transmission. For numerical results we consider an $8 \times 8$ MIMO system transmitting $M=8$ data streams. The channel values are generated as realizations of a random matrix with complex Gaussian entries of $C N(0,1)$ with an exponential correlation matrix $\left\{(\mathbf{R})_{i k}=(0.9)^{|i-k|} e^{j 2 \pi(i-k) / 12}\right\}$ [15]. The AWGN variance used in the simulations is 0.1 .

First, we consider a system with a power constraint $P_{s} \leq 8$. For the system considered, we have compared the performance of the linear transceiver structure we proposed (TX-RX1), the linear transceiver structure using the ML estimate of the channel without considering the channel estimation error (TXRX2), and their VBLAST versions [16]: VBLAST transmission with MMSE receivers considering the channel estimation error (TX-RX3), and VBLAST transmission with MMSE receiver using the ML estimate of the channel (TX-RX4). For the sake of a fair comparison, linear MMSE receivers are used for VBLAST detection. We plot, in Fig. 1 and 2, the total MSE and the BER performances achieved by each linear transceiver structure versus the channel estimation error variance, $\sigma_{e}^{2}$. The results are evaluated over 10000 realizations of the MIMO channel with the same channel estimate. The linear transceiver structure we proposed, TX-RX1 performs the best in terms of both MSE and BER, and precoding and considering the channel estimation error provide robustness against the channel uncertainty. When the channel estimation error is low, the total MSE and BER performances of TXRX1 and TX-RX2 pairs and TX-RX3 and TX-RX4 are very close as expected. However, as the accuracy of the channel estimate gets worse, the performances of the linear transceivers using the ML channel estimate, TX-RX2 and TX-RX4 suffer dramatically, whereas the receiver structures considering the channel estimation errors, TX-RX1 and TX-RX3 provide 


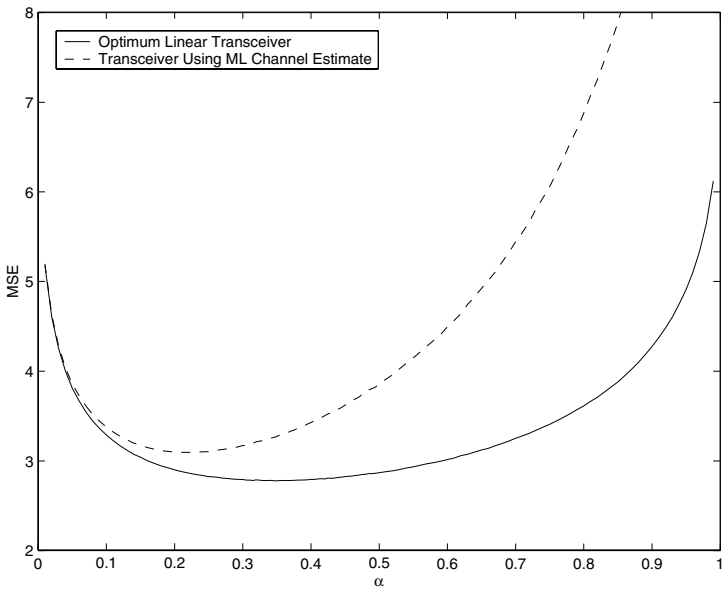

Fig. 3. MSE vs $\alpha$ for $8 \times 8$ MIMO system with $L_{d}=2$ and $P_{\text {total }}=50$

robustness against the channel estimation errors.

To investigate the effect of the power allocation among the channel estimation process and data transmission, we consider a system with two different values of $L_{d}$. We consider a system with $L_{d}=2,40$ and $P_{\text {total }}=50,150$. We evaluate the total MSE over 10000 realizations of the MIMO channel for different power allocation schemes. Fig. 3 and 4 show the effect of power allocation on the total MSE as $\alpha$ changes for both the optimal linear transceiver we propose and the linear transceiver structure using ML channel estimate for $L d=2$ and $L d=40$ cases respectively. It is observed that the minimum total MSE is achieved at the optimal power allocation. For $L_{d}=2 \alpha_{o p t}=0.35$, and it is in the interval $\left[\alpha_{\min }, \alpha_{\max }\right]=[0.334,0.354]$. The optimum power allocation for uncorrelated case, $\tilde{\alpha}$ is 0.335 . For the second case, $N_{T}<$ $L_{d}=40$ and $P_{\text {total }}=150, \alpha_{\text {opt }}=0.67 \in\left[\alpha_{\text {min }}, \alpha_{\max }\right]=$ $[0.658,0.691]$ with $\tilde{\alpha}=0.689$. Observe that the effect of power allocation on the total MSE is not much sensitive when the optimal linear transceiver structure is used. However, the best performance is achieved when the system is optimized in terms of both the power allocation and linear transceiver structure. For both cases, optimum power allocation with the optimal linear transceiver achieves the minimum total MSE.

\section{CONCLUSIONS}

In this paper, we have developed the optimum linear transceiver structure for a MIMO link with arbitrary correlation among the receive antennas, that minimizes the total MSE in the presence of channel estimation errors, and distributes the total MSE equally among the parallel data streams. Motivated by the profound effect of the quality of channel estimation on the performance of the MIMO link, we have considered the problem optimum sharing of resources between the process of channel estimation and data transmission. Considering the total power as the limited resource, we have showed that, given the coherence time of the channel, there is a unique solution to the optimum allocation problem between the training based ML channel estimation and data transmission, and that the solution differs from the previous results reported for

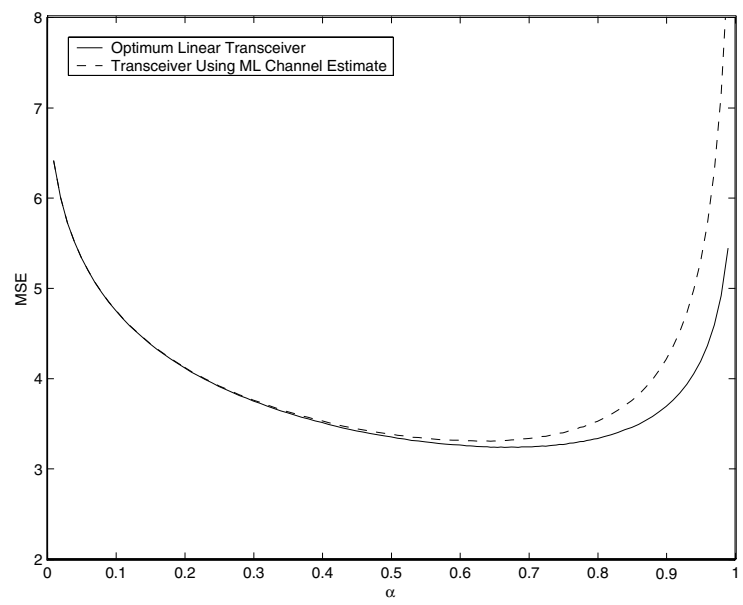

Fig. 4. MSE vs $\alpha$ for $8 \times 8$ MIMO system with $L_{d}=40$ and $P_{\text {total }}=150$ uncorrelated MIMO channels. We observe that the optimum power allocation depends on the system parameters, and the correlation structure of the receiver antennas.

\section{REFERENCES}

[1] E. Telatar. Capacity of multi-antenna Gaussian channels. European Tran. on Telecommunications, 10(6):585 - 595, Nov./Dec. 1999.

[2] V. Tarokh, N. Seshadri, and R. Calderbank. Space-time codes for high data rate wireless communication: performance criterion and code construction. IEEE Tran. on Info. Theory, 44(2):744-765, March 1998.

[3] G. J. Foschini. Layered space-time architecture for wireless communication in a fading environment when using multi-element antennas. Bell Labs Technical Journal, 1(2):41-59, Autumn 1996.

[4] B. Hassibi and B. M. Hochwald. How much training is needed in multiple antenna wireless links? IEEE Tran. on Info. Theory, 48(4):951 - 963, April 2003.

[5] H. Sampath, P. Stoica, and A. Paulraj. Generalized linear precoder and decoder design for MIMO channels using the weighted MMSE criterion. IEEE Tran. on Comm., 49(12):2198-2206, Dec. 2001.

[6] J. H. Kotecha and A. M. Sayeed. Transmit signal design for optimal estimation of correlated MIMO channels. IEEE Tran. on Signal Processing, 52(2):546 - 557, Feb. 2004.

[7] H. Sampath and A. Paulraj. Linear precoding for space-time coded systems with known fading correlations. IEEE Comm. Letters, 6(6):239 - 241, June 2002.

[8] S. A. Jafar and A. Goldsmith. Transmitter optimization and optimality of beamforming for multiple antenna systems. IEEE Tran. on Wireless Comm., 3(4):1165 - 1175, July 2004.

[9] D. P. Palomar, M. A. Lagunas, and J. M. Cioffi. Optimum linear joint transmit-receive processing for MIMO channels with QoS constraints. IEEE Tran. on Signal Processing, 52(5):1179-1197, May 2004.

[10] S. Serbetli, S. Bethanabhotla, and A. Yener. The effect of channel estimation on transceiver design for MIMO systems with QoS constraints. In Conference on Info. Sciences and Systems, CISS'04, March 2004.

[11] S. Serbetli and A. Yener. Transceiver optimization for multiuser MIMO systems. IEEE Tran. on Signal Processing, 52(1):214-226, Jan. 2004.

[12] S. Zhou and G. B. Giannakis. Optimal transmitter eigen-beamforming and space time block coding based on channel mean feedback. IEEE Tran. on Signal Processing, 50(10):2599-2613, October 2002.

[13] S. Serbetli and A. Yener. MMSE transmitter design for correlated MIMO systems with imperfect channel estimates: Power allocation trade-offs. submitted to IEEE Trans. on Wireless Comm., August 2004. http://labs.ee.psu.edu/faculty/yener/publications.html.

[14] D. Bertsekas. Nonlinear Programming. Athena Scientific, 1995.

[15] S. L. Loyka. Channel capacity of MIMO architecture using the exponential correlation matrix. IEEE Comm. Letters, 5(9):369 - 371, Sept. 2001.

[16] G. D. Golden, C. J. Foschini, R. A. Valenzuela, and P. W. Wolniansky. Detection algorithm and initial laboratory results using V-BLAST spacetime communication architecture. Electronics Letters, 35(1):14-16, Jan. 1999. 